\title{
Detection of residual metastatic tumor in the brain following Gamma Knife radiosurgery using a single or a series of magnetic resonance imaging scans: An autopsy study
}

\author{
MADOKA SAKURAMACHI ${ }^{1}$, HIROSHI IGAKI ${ }^{2}$, MASAKO IKEMURA ${ }^{3}$, HIDEOMI YAMASHITA ${ }^{1}$, \\ KAE OKUMA $^{1}$, NORIYASU SEKIYA ${ }^{1}$, YAYOI HAYAKAWA ${ }^{4}$, AKIRA SAKUMI ${ }^{5}$, WATARU TAKAHASHI ${ }^{1}$, \\ HIROTAKA HASEGAWA $^{6}$, MASASHI FUKAYAMA ${ }^{3}$ and KEIICHI NAKAGAWA ${ }^{1}$ \\ ${ }^{1}$ Department of Radiology, The University of Tokyo Hospital, Tokyo $113-8655 ;{ }^{2}$ Department of \\ Radiation Oncology, National Cancer Center Hospital, Tokyo 104-0045; ${ }^{3}$ Department of Pathology, \\ The University of Tokyo Hospital, Tokyo 113-8655; ${ }^{4}$ Department of Radiology, New Tokyo Hospital, Matsudo, \\ Chiba 271-2232; ${ }^{5}$ Department of Radiation Therapy, NTT Medical Center Tokyo, Tokyo 141-0022; \\ ${ }^{6}$ Department of Neurosurgery, The University of Tokyo Hospital, Tokyo 113-8655, Japan
}

Received July 20, 2016; Accepted March 24, 2017

DOI: $10.3892 / 01.2017 .6359$

\begin{abstract}
The aim of the present study was to investigate the usefulness of magnetic resonance image (MRI) for the detection of residual tumors following Gamma Knife radiosurgery (GKR) for brain metastases based on autopsy cases. The study investigated two hypotheses: i) Whether a single MRI may detect the existence of a tumor; and ii) whether a series of MRIs may detect the existence of a tumor. The study is a retrospective case series in a single institution. A total of 11 brain metastases in 6 patients were treated with GKR between 2002 and 2011. Histopathological specimens from autopsy were compared with reconstructed follow-up MRIs. The maximum diameters of the lesions on MRI series were measured, and the size changes classified. The primary sites in the patients were the kidneys $(n=2)$, lung $(n=1)$, breast $(n=1)$ and colon $(n=1)$, as well as 1 adenocarcinoma of unknown origin. The median prescribed dose for radiosurgery was $20 \mathrm{~Gy}$ (range, 18-20 Gy),
\end{abstract}

Correspondence to: Dr Madoka Sakuramachi, Department of Radiology, The University of Tokyo Hospital, 7-3-1 Hongo, Bunkyo, Tokyo 113-8655, Japan

E-mail: sakuramachim-rad@h.u-tokyo.ac.jp

Abbreviations: GKR, Gamma Knife radiosurgery; Gd-MRI, gadolinium-enhanced magnetic resonance imaging; WBRT, whole brain radiotherapy; T1WI, T1-weighted imaging; T2WI, T2-weighted imaging; FLAIR, fluid-attenuated inversion recovery; DWI, diffusion-weighted imaging; FDG-PET, fluorodeoxyglucose-positron emission tomography; PR, partial response; $\mathrm{PD}$, progressive disease; $\mathrm{SD}$, stable disease; $\mathrm{BBB}$, blood-brain barrier; NSCLC, non-small-cell lung cancer; BED, biological effective dose

Key words: brain metastases, Gamma Knife radiosurgery, follow-up magnetic resonance imaging, pathology, autopsy and median time interval between GKR and autopsy was 10 months (range, 1.6-20 months). The pathological outcomes included 7 remissions and 4 failures. Enhanced areas on gadolinium-enhanced MRI contained various components: Viable tumor cells, tumor necrosis, hemorrhage, inflammation and vessels. Regarding the first hypothesis, it was impossible to distinguish pathological failure from remission with a single MRI scan due to the presence of various components. Conversely, in treatment response (remission or failure), on time-volume curves of MRI scans were in agreement with pathological findings, with the exception of progressive disease in the acute phase (0-3 months). Thus, regarding the second hypothesis, time-volume curves were useful for predicting treatment responses. In conclusion, it was difficult to predict treatment response using a single MRI, and a series of MRI scans were required to detect the existence of a tumor.

\section{Introduction}

The incidence of brain metastases may be increasing, due to both improved detection of small metastases by magnetic resonance imaging (MRI) and improved control of extracerebral disease as a result of improved systematic therapy $(1,2)$. Whole brain radiotherapy (WBRT) and Gamma Knife radiosurgery (GKR) are standard modalities of treatment for brain metastases. WBRT has been a classical treatment for almost all cases of brain metastases. GKR has been performed for a limited number and small sizes of brain metastases $(\leq 4$ and $<3 \mathrm{~cm}$ in diameter) $(3,4)$, but recent multiple randomized trials support the use of GKR in the initial management of patients with $<5$ brain metastases (5). GKR provides a high control rate due to spatially accurate and high-conformal isodose curve to the target. However, the recurrence of brain metastases has been identified to occur in $10-16 \%$ of lesions following treatment with GKR (5-9). Tumor recurrence usually presents with a progressive increase in lesion size on gadolinium-enhanced MRI (Gd-MRI) (10-13). Certain enlargements are caused by 
tumor progression, whereas others are caused by radiation effects without tumor progression. It is often difficult to distinguish radiation effects from tumor progression. Pathological examination, including surgical resection or biopsy, is performed to predict the cause of enlarged lesion size on MRI scans. Although there are numerous studies (14-17) comparing the pathological findings from surgical resection or biopsy with MRI findings, this method may not always be accurate because of the uncertain location information. Investigations comparing pathological findings from autopsy with MRI findings is required to acquire accurate interpretations of MRI.

The aim of the present study was to investigate the usefulness of MRI for the detection of residual tumors following GKR for brain metastases. Two hypotheses were investigated: i) Whether a single MRI may detect tumor existence; and ii) whether a series of MRIs may detect tumor existence. Follow-up MRI and pathological results in brain metastases were compared using autopsy cases to answer these questions.

\section{Materials and methods}

Study design. This study was a retrospective case series performed in a single institution; The University of Tokyo Hospital (Tokyo, Japan).

Ethics statement. The study protocol was approved by the ethical review board of the University of Tokyo Hospital (authorization no. 10857). Written informed consent was acquired from patients in advance, or from surrogates following mortality.

Patients. Patients with metastatic tumors in the brain, who were treated with GKR and received autopsy with craniotomy between August 2003 and April 2011 at The University of Tokyo Hospital were included. Patients with brain metastases without follow-up MRIs were excluded. The characteristics of the included patients are summarized in Table I.

Radiation techniques. All lesions were treated with GKR. A Leksell frame (Elekta Instruments AB, Stockholm, Sweden) was attached to secure the head of the patient in place. Subsequently, contrast-enhanced stereotactic MRI was performed to obtain precise data of the $3 \mathrm{D}$ coordinates of the tumors. The Leksell Gamma Plan (Elekta Instruments AB) was used to plan the treatment for tumors by stereotactic Gd-MRI and thin-slice diagnostic Gd-MRI. Treatments were delivered using the Leksell Gamma Knife B or 4C (Elekta Instruments $\mathrm{AB}$ ). A dose of $20 \mathrm{~Gy}$ was prescribed for patients in whom GKR was the initial radiotherapy, and $18 \mathrm{~Gy}$ for patients who had undergone WBRT prior to GKR. The appropriate isodose curves were calculated considering the shape of the target tumor. Target volumes were defined based on the area of contrast enhancement plus a margin of $1 \mathrm{~mm}$. For each patient, the recommendation for WBRT was based on the attending physician's discretion, using a linear accelerator with a median prescribed dose of $30 \mathrm{~Gy}$ in 3-Gy fractions or $40 \mathrm{~Gy}$ in 2-Gy fractions.

Follow-up MRI and other modalities. Patients received follow-up MRIs every 1-3 months following GKR to detect recurrence and/or newly occurring tumors. Routine MRI sequences included axial T1-weighted imaging (T1WI), axial T2-weighted imaging (T2WI), coronal fluid-attenuated inversion recovery (FLAIR), coronal and axial Gd-MRI, axial diffusion-weighted imaging (DWI), and axial apparent diffusion coefficient. Fluorodeoxyglucose-positron emission tomography (FDG-PET) was performed to distinguish tumor recurrence from radiation effects in an enlarged lesion, or to perform systemic investigation for the patient with the adenocarcinoma of unknown origin.

Autopsyand histological examination. Autopsy was performed within $24 \mathrm{~h}$ after mortality. Brain sections were fixed in $20 \%$ neutral-buffered formaldehyde for 2-4 weeks. The cerebrum was cut into coronal slices 7-10-mm thick. The specimens were cut into small quadrangular pieces and embedded in paraffin, then cut again to produce $4-6-\mu$ m-thick paraffin-embedded tissue sections. The specimens were mounted on glass microscope slides coated with $0.01 \%$ poly-L-lysine, and maintained at $37^{\circ} \mathrm{C}$ in an incubator overnight. Subsequent to dehydration with an alcohol and xylene series, hematoxylin staining ( $2 \min 30 \sec x 3)$ and eosin staining $(2 \min x 1)$ at room temperature was performed for primary examination. Sections from formalin-fixed paraffin-embedded tissue blocks were subjected immunohistochemical analysis using a Ventana BenchMark XT automated immunostainer (Roche Diagnostics, Basel, Switzerland). Staining conditions were as follows; samples were incubated with primary antibody incubation for $30 \mathrm{~min}$ at room temperature, following addition of $\mathrm{CC} 1$ standard (CC1-buffer, $\left.60 \mathrm{~min}, 95^{\circ} \mathrm{C}\right)$. The applied primary antibody was Ki-67 (cat. no. M7240; Clone MIB-1; dilution, 1:200; Dako; Agilent Technologies, Inc., Santa Clara, CA, USA). Immunohistochemical results of Ki-67 were evaluated for identifying tumor proliferation. Pathological failure was defined as the detection of Ki-67-positive tumor cells by histological examination in treated brain metastases of autopsy specimens.

Analysis. First, the most recent MRI scans and the pathological results from autopsy were compared. The MRI scans were reconstructed using a free 3D imaging program, OsiriX (Pixmeo, Geneva, Switzerland), to adapt the slices of the scans to the cut slices of brain at autopsy. The last single MRI was compared with pathological specimen and pathological results in the gadolinium-enhanced area were obtained. Subsequently, the maximum diameters of the lesions were measured on a series of Gd-MRI scans, the percentage of the tumor diameter was calculated, referring to the diameter of the lesion at the time of GKR as baseline, and a time-volume curve was produced. The treatment responses of the tumors were classified as follows: Complete response (CR), disappearance of target lesion; partial response (PR), $\geq 30 \%$ decrease in the diameter of the target lesion, taking as reference the baseline diameter; progressive disease (PD), $\geq 20 \%$ increase in the diameter of the target lesion, taking as reference the baseline diameter; stable disease (SD), neither sufficient shrinkage to qualify for PR nor sufficient increase to qualify for PD, taking as reference the baseline diameter. The follow-up period following GKR was divided into three phases: Acute (0-3 months); sub-acute (3-6 months); and late ( $>6$ months). 


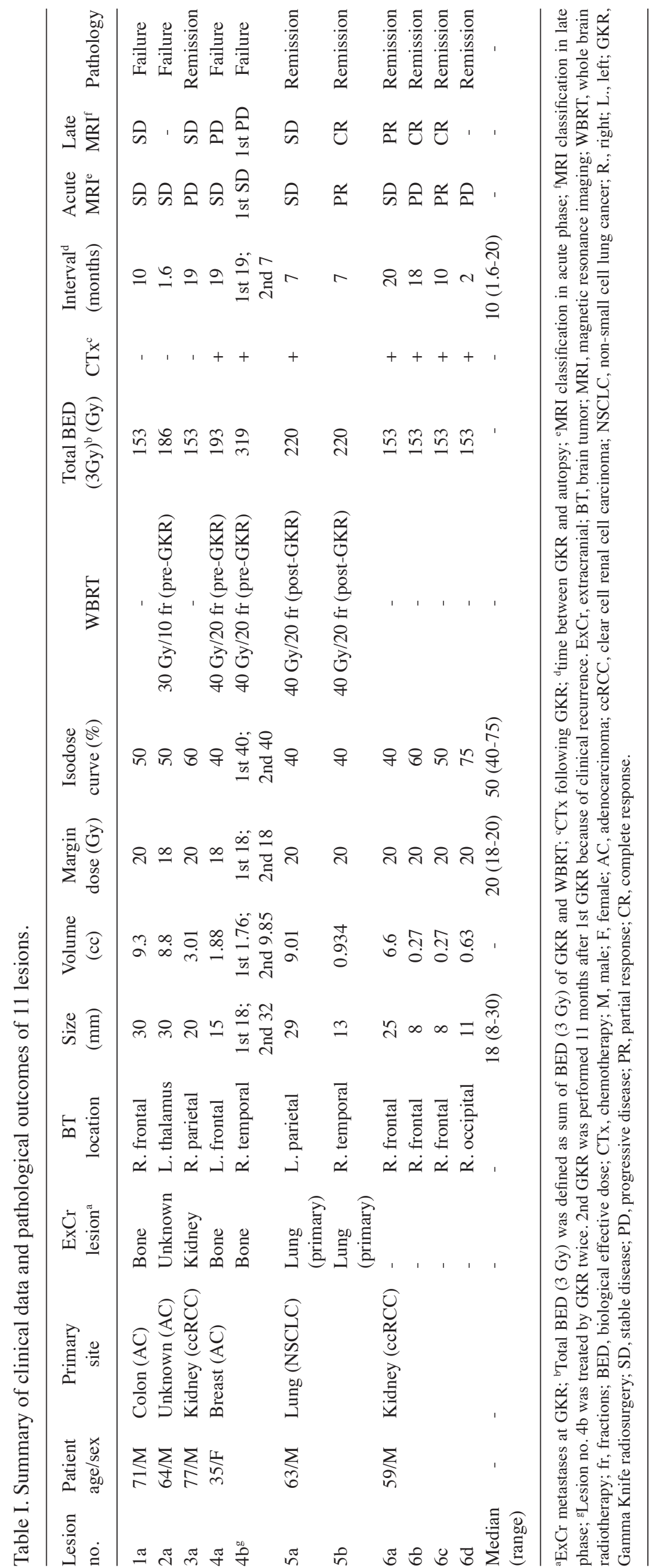


Treatment responses were categorized by the size changes (CR, PR, SD or PD) and the follow-up period (acute or late phases). In the present study, 'temporary enlargement' was defined an initial growth of $>20 \%$, and reduction to less than the baseline size within 3 months. To assess the total radiation effect of GKR and WBRT, the total biological effective dose (BED; 3 Gy) of the tumor margins was calculated. The present study presumed that BED ( $3 \mathrm{~Gy})$ reflected late biological effect from radiation therapy.

\section{Results}

Patient characteristics and treatments. A total of 9 patients with 14 metastatic lesions in the brain were treated with GKR and received autopsy with craniotomy between June 1995 and June 2013 at The University of Tokyo Hospital. Brain metastases without follow-up MRIs were excluded. As a result, 6 patients with a total of 11 brain metastases were eligible for the present study. These lesions were treated with GKR between October 2002 and February 2011.

The median age at diagnosis was 63.5 years (range, 35-77 years). The male:female ratio was 5:1. Sites of primary tumors were the kidneys in 2 patients, lung in 1 patient, breast in 1 patient, colon in 1 patient and an adenocarcinoma of unknown origin in 1 patient. The numbers of brain metastases at diagnosis were 1 tumor in 3 patients, 2 tumors in 2 patients, and 4 tumors in 1 patient, respectively. WBRT was performed in 3 patients prior or subsequent to GKR. Surgery was performed in 1 patient prior to GKR. The median follow-up time was 15 months (range, 1.6-20 months). None of the patients succumbed with any clear evidence of neurological symptoms. Table I summarizes the clinical data and pathological outcomes. A total of 11 brain metastases in 6 patients were treated with GKR. The median prescribed dose was $20 \mathrm{~Gy}$ (range, 18-20 Gy) at the tumor margin, with a median maximal dose of 40 Gy (range, 27-50 Gy). GKR was performed twice in the same lesion in 1 brain metastasis from breast cancer (lesion no. $4 \mathrm{~b}$ ).

The pathological outcomes were 7 remissions and 4 failures. The pathological outcomes of the all lesions were the same in every patient: Either all remissions or all failures. Although 1 lesion (no. 4b) received the highest total BED (3 Gy) of 319 Gy due to repeated GKR, it did not exhibit radiation necrosis.

Comparison of the last MRI results with pathological results. Table II summarizes the associations between the details of the last MRI and pathological results. A case of pathological failure is presented in Fig. 1 (lesion no. 1a). The contrast-enhanced areas on Gd-MRI contained various pathological components, including viable tumor cells, tumor necrosis, hemorrhage, inflammation and vessels. The degree of contrast on Gd-MRI was similar in all components. All viable tumor tissue and hemorrhages demonstrated contrast enhancement. Some areas of tumor necrosis, inflammation and vessels exhibited contrast enhancement, while others did not.

Time-volume curves from a series of MRI scans. Time-volume curves determined by Gd-MRI are shown in Fig. 2, a summary of which is listed in Table III. PD indicated pathological failures in the late phase. Treatment response, remission or failure and

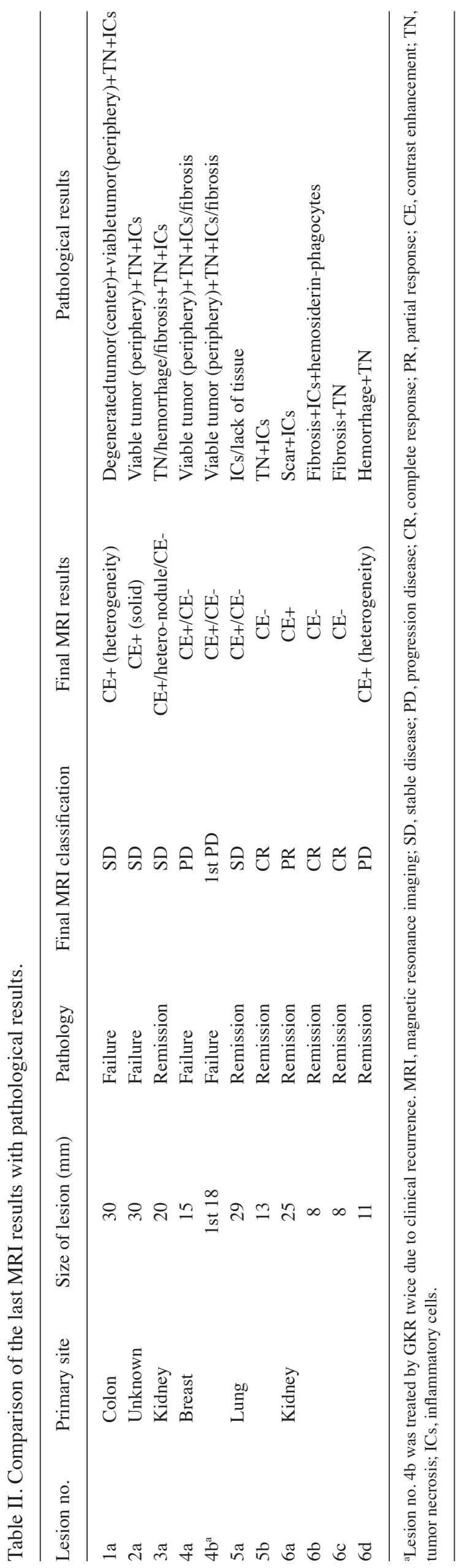




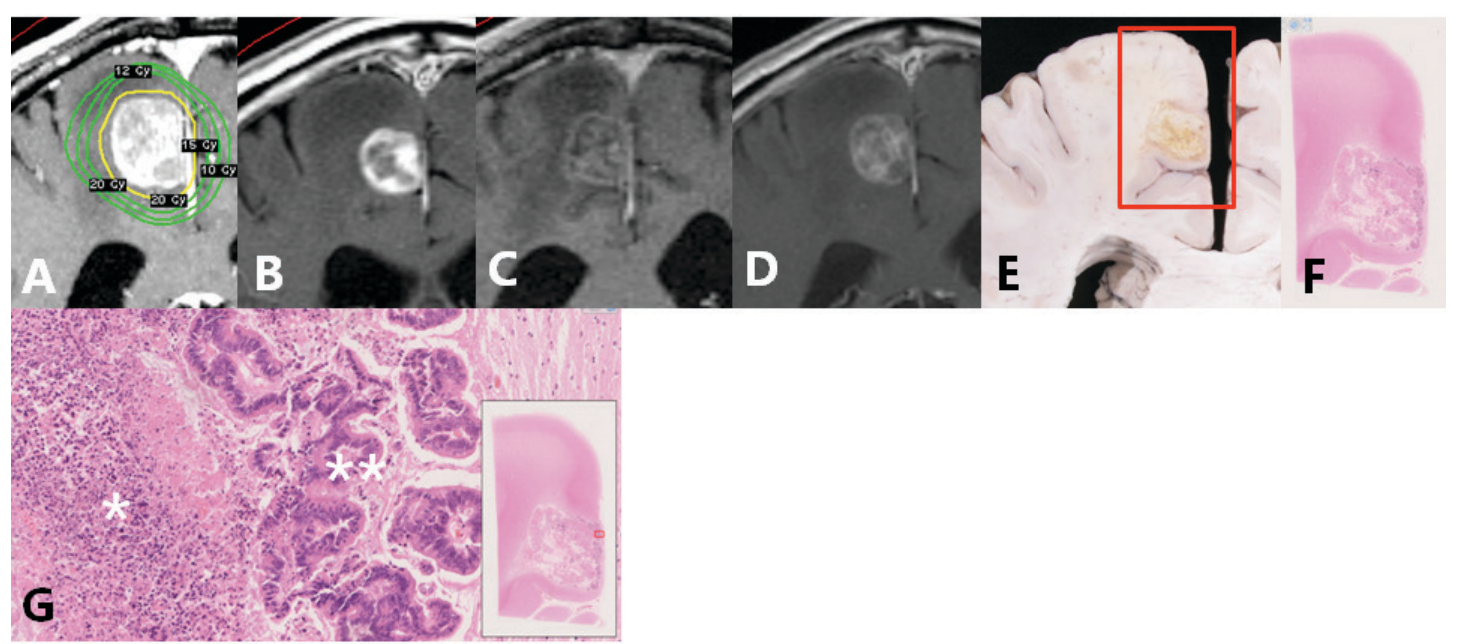

Figure 1. Images and pathological findings of one case (lesion no. 1a). (A) Dose distribution at GKR. (B) Gd-MRI 1 month following GKR. (C) Gd-MRI 3 months after GKR, classified as SD in acute phase. (D) final Gd-MRI 7 months after GKR, classified as SD in late phase. (E) Macroscopic view at autopsy 10 months after GKR. (F) Histological examination. (G) "Tumor necrosis and ***iable tumor cells were observed at the periphery and center of the lesion, respectively (magnification, 20x10). GKR, Gamma Knife radiosurgery; Gd-MRI, gadolinium-enhanced magnetic resonance imaging; SD, stable disease.

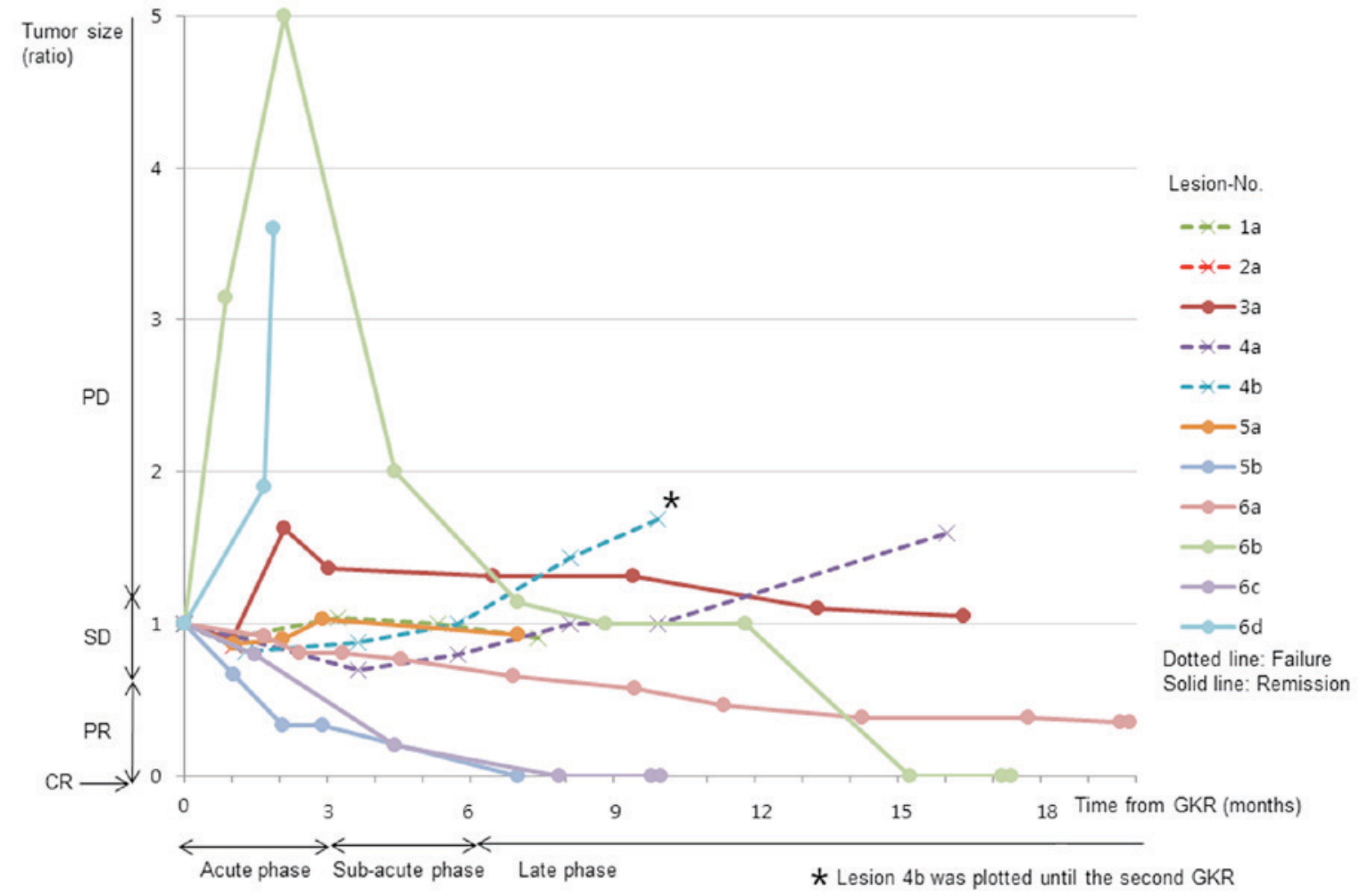

Figure 2. Time-volume curves on Gd-MRI for 11 lesions. The maximum diameters of the lesions were measured on a series of Gd-MRI scans, the percentage of the tumor diameter was calculated, referring to the diameter of the lesion at the time of GKR as baseline, and a time-volume curve was produced. Pathological failures are shown by dotted lines and pathological remissions are shown by solid lines. GKR, Gamma Knife radiosurgery; Gd-MRI, gadolinium-enhanced magnetic resonance imaging; PD, progressive disease; SD, stable disease; PR, partial response; CR, complete response.

on time-volume curves of MRI scans were in agreement with pathological findings, excluding acute PD lesions (Table II; lesion nos. 3a, 6b and 6d). A total of 2 acute PD lesions (nos. 6b and 6d) demonstrated high intensity on non-enhanced T1WI, indicating hemorrhage. Histopathological examination revealed hemosiderin-phagocytes (lesion no. 6b) and hemorrhages (lesion no. 6d) without viable tumor tissue. A partially enlarged, high-intensity area on Gd-MRI was observed in 1 acute PD lesion (no. 3a). The enlarged area on Gd-MRI was iso-intense on T2WI, indicating an expansion of contrast enhancement, and not hemorrhage. As the enlarged area on Gd-MRI reduced in size within 3 months, enlargement was judged to be temporary. Histopathological examination indicated only edema in the temporarily enlarged area. Certain SD lesions (nos. 1a and 2a) demonstrated remnant viable tumor, whereas others (nos. 3a and 5a) did not in either the acute 
Table III. Association between MRI classification and pathological outcomes.

\begin{tabular}{lccc}
\hline MRI classification & $\begin{array}{c}\text { Pathological } \\
\text { remission }\end{array}$ & $\begin{array}{c}\text { Pathological } \\
\text { failure }\end{array}$ & Total \\
\hline Acute phase & 0 & 0 & 0 \\
CR & 2 & 0 & 2 \\
PR & 2 & 4 & 6 \\
SD & 3 & 0 & 3 \\
PD & & & \\
Late phase & 3 & 0 & 3 \\
CR & 1 & 0 & 1 \\
PR & 2 & 1 & 3 \\
SD & 0 & 2 & 2 \\
PD & 1 & 1 & 2 \\
No follow-up & & & \\
until late phase & 7 & 4 & 11 \\
Total & & & \\
\hline
\end{tabular}

MRI, magnetic resonance imaging; CR, complete response; PR, partial response; $\mathrm{SD}$, stable disease; $\mathrm{PD}$, progression disease.

Table IV. Results of DWI, FLAIR, and FDG-PET scans.

\begin{tabular}{lccr}
\hline $\begin{array}{l}\text { Scan } \\
\text { findings }\end{array}$ & $\begin{array}{c}\text { Pathological } \\
\text { remission }\end{array}$ & $\begin{array}{c}\text { Pathological } \\
\text { failure }\end{array}$ & Total \\
\hline DWI & & 3 & 10 \\
$\quad$ Normal intensity & 7 & 1 & 1 \\
Abnormal intensity & 0 & & \\
FLAIR & & 2 & 9 \\
Stable & 7 & 2 & 2 \\
Enlarged peritumoral & 0 & & \\
high intensity & & 1 & 1 \\
FDG-PET & & 2 & 2 \\
Normal uptake & 0 & 1 & 8 \\
Increased uptake & 0 & & \\
No image & 7 & &
\end{tabular}

Values indicate number of lesions. DWI, diffusion-weighted imaging; FLAIR, fluid-attenuated inversion recovery; FDG-PET, fluorodeoxyglucose-positron emission tomography.

or the late phase. Late PD lesions (nos. 4a and 4b) exhibited tumor recurrences (no. 4b).

Other sequences and modalities. Regarding DWI, FLAIR and FDG-PET scans, the examination outcomes are summarized in Table IV. From the results of the DWI scans, only 1 lesion (no. 2a) exhibited abnormal intensity among the 4 pathological failures (nos. 1a, 2a, 4a and 4b). As for FLAIR, enlarged peritumoral high intensity was observed in 2 lesions (nos. 4a and 4b) among the 4 pathological failures. Concerning
FDG-PET, increased uptake was observed in 2 lesions (nos. 2a and 4 b) but not in 1 lesion (no. 4a) among the 4 pathological failures.

\section{Discussion}

To the best of our knowledge, the present study is the first to report a precise correlation between MRI and pathological results in autopsy cases of brain metastases treated with GKR. In previous studies, correlations between MRI and pathology using surgical or biopsy specimens were studied while relying on insufficient orientation; the locations of the MRI features were not adapted to the location of pathological features directly $(15,18)$. To resolve this problem, the slices of the reconstructed MRIs were adapted to the macroscopically viewed slices of the brain in autopsy cases. This enabled the correct study of the correlations between MRI and pathological findings. The treatment method of GKR was standardized, making it easy to compare its effects. At various timings from acute to late phase, the histopathological outcomes were evaluated.

Although pathological failure was evident in 4/11 lesions (36\%), no patient succumbed to brain metastases during the course of the present study. It is often hypothesized that pathological confirmation of remnant viable tumors by surgical specimen may be an overdiagnosis in view of the clinical course $(15,19)$.

To investigate the usefulness of MRI for the detection of residual tumor following GKR for brain metastases, two hypotheses were investigated: i) Whether a single MRI scan may detect tumor existence; and ii) whether a series of MRI scans may detect tumor existence.

According to certain studies $(20,21)$, contrast enhancement in the central nervous system is a combination of two primary processes: Vascular (intravascular) enhancement and interstitial (extravascular) enhancement. New blood vessels (angiogenesis), active inflammation (infectious and noninfectious), cerebral ischemia and pressure overload (eclampsia and hypertension) are known as extravascular enhancement. All of these changes are associated with alterations in permeability of the blood-brain barrier (BBB). In the present study, five pathological components were observed: Viable tumor cells, tumor necrosis, hemorrhage, inflammation and vessels in the area of contrast enhancement on Gd-MRI. There were abundant tumor vessels adjacent to viable tumor tissue and tumor necrosis. $\mathrm{BBB}$ of tumor vessels were vulnerable. Hemorrhage indicated injury of the vessels, and inflammation occasionally indicated active inflammation. As all of these components were associated with alterations in BBB permeability, contrast enhancement itself was not useful for distinguishing viable tumor tissues from the other components. Other MRI sequences, such as DWI and FLAIR, were not always useful. Thus, regarding the first hypothesis, a single MRI was not able to assess the existence of viable tumor tissue.

Of 3 acute PD lesions, 2 (lesion nos. 6b and 6d) were confirmed as hemorrhage by histopathology. These two lesions were metastases from renal cell carcinoma. Brain metastases, particularly from renal cell carcinoma, tend to exhibit bleeding as part of the natural course of the disease $(22,23)$, and bleeding was identified in $9 \%$ of patients following GKR 
in a previous study (24). Bleeding at the tumor site is not a rare event following stereotactic irradiation in renal cell carcinoma.

The third acute PD lesion (no. 3a) exhibited temporary high-intensity enlargement on Gd-MRI, which demonstrated iso-intensity on T1WI/T2WI. The histopathology indicated that the temporarily enlarged area consisted only of edema. Several previous studies $(25,26)$ demonstrated temporary enlargement of brain metastases following stereotactic radiosurgery: One study reported a rate of $12 \%$ (11/87 cases) and a median duration of 3 months (range, 2-6 months) (25); while the other study reported temporary enlargement in 7\% (5/73 cases) of all lesions following GKR (26). However, the detailed mechanism of temporary enlargement is not clear. It was hypothesized that extravascular enhancement by vessel changes and consequential increased BBB permeability caused temporary, enhancing enlargement on MRI. Park et al (27) suggested that endothelial cells were injured and the permeability of the vessels rose to a peak within $24 \mathrm{~h}$ after irradiation when normal vessels were irradiated. This increased permeability then remained for $\sim 1$ month. The magnitude of these changes depended on the radiation dose. An increase in the permeability of vessels led to invasion of inflammatory cells and fibroblasts, facilitating fibrosis (22). Similar changes may have occurred in 1 patient of the present study (no. 3a). As chronic changes of normal vessels, such as increased wall thickness, were observed in the area of temporary enlargement, these normal vessels were assumed to have presented increased permeability as a result of radiation injury in the acute phase. Following these acute changes, permeability of these normal vessels would decrease with increasing wall thickness.

In the present study, 2 acute PR lesions (nos. 5b and 6c) continued to shrink, becoming CR on the last MRI in the late phase, and these lesions were confirmed as pathological remission. Da Silva et al (28) identified that tumors with a histology associated with high radiosensitivity, such as breast cancer and non-small-cell lung cancer (NSCLC), tended to shrink in the acute phase. In the present study, acute shrinkage was observed in the metastases that arose from NSCLC (lesion no. 5b) and renal cell carcinoma (lesion no. 6c). Lesions from breast cancer were reduced by a small amount, but were diagnosed as SD. Tumor necrosis, inflammation and hemorrhages occurred in the acute or late phases, and exhibited contrast enhancement in the absence of viable tumor tissues.

The time-volume curves demonstrated a good association with pathological outcomes, excluding acute PD. The determination of acute PD was due to hemorrhage or temporary enlargement on Gd-MRI. Acute PD lesions decreased in size during the late phase, indicating that they should be observed for an extended period. In addition, tumor necrosis should be monitored in the late phase. There was no case of radiation necrosis in the present study, but generally it is difficult to distinguish radiation necrosis from tumor recurrence by MRI results in late PD lesions (15). Thus, as regards the second hypothesis, a series of MRI scans may detect the presence of residual tumor. For acute PD, subsequent time-volume curves were useful to distinguish pathological remission from failure.

High intensity on DWI, enlarged size of high intensity on FLAIR, and increased uptake on FDG-PET were occasionally useful for detecting tumor recurrence, but the absence of these signs did not always rule out tumor existence.
The present study was subject to certain limitations. First, it comprised a small number of patients, and more patients are required to confirm the findings. In addition, there was a selection bias due to the use of only autopsy cases. Furthermore, there was a delay between the last MRI and autopsy, and the MRI results may change within that interval. Additionally, there was no patient with radiation necrosis included in the present study.

It may be concluded that time-volume curves from a series of MRI scans are useful for predicting pathological tumor responses. Particularly for acute PD lesions exhibiting hemorrhage or temporary enlargement, subsequent time-volume curves were useful to distinguish pathological remission from failure. It is impossible to distinguish pathological failure from remission with a single MRI. This is because contrast enhancement on Gd-MRI contained viable tumor tissues, tumor necrosis, hemorrhage, inflammation and vessels.

\section{Acknowledgements}

The authors gratefully acknowledge the work of members of Department of Pathology and Radiology of The University of Tokyo Hospital. The authors would also like to thank Mr. Gerz for careful English proofreading.

\section{References}

1. Wen PY and Loeffler JS: Management of brain metastases. Oncology (Williston Park) 13: 941-954, 957-962, 969, 1999.

2. Bouffet E, Doumi N, Thiesse P, Mottolese C, Jouvet A, Lacroze M, Carrie C, Frappaz D and Brunat-Mentigny M: Brain metastases in children with solid tumors. Cancer 79: 403-410, 1997.

3. Andrews DW, Scott CB, Sperduto PW, Flanders AE, Gaspar LE, Schell MC, Werner-Wasik M, Demas W, Ryu J, Bahary JP, et al: Whole brain radiation therapy with or without stereotactic radiosurgery boost for patients with one to three brain metastases: Phase III results of the RTOG 9508 randomised trial. Lancet 363: 1665-1672, 2004.

4. Kocher M, Soffietti R, Abacioglu U, Villà S, Fauchon F, Baumert BG, Fariselli L, Tzuk-Shina T, Kortmann RD, Carrie C, et al: Adjuvant whole-brain radiotherapy versus observation after radiosurgery or surgical resection of one to three cerebral metastases: Results of the EORTC 22952-26001 study. J Clin Oncol 29: 134-141, 2011.

5. Yamamoto M, Serizawa T, Shuto T, Akabane A, Higuchi Y, Kawagishi J, Yamanaka K, Sato Y, Jokura H, Yomo S, et al: Stereotactic radiosurgery for patients with multiple brain metastases (JLGK0901): A multi-institutional prospective observational study. Lancet Oncol 15: 387-395, 2014

6. Young RF: Radiosurgery for the treatment of brain metastases. Semin Surg Oncol 14: 70-78, 1998.

7. Mori Y, Kondziolka D, Flickinger JC, Logan T and Lunsford LD: Stereotactic radiosurgery for brain metastasis from renal cell carcinoma. Cancer 83: 344-353, 1998.

8. Mori Y, Kondziolka D, Flickinger JC, Kirkwood JM, Agarwala S and Lunsford LD: Stereotactic radiosurgery for cerebral metastatic melanoma: Factors affecting local disease control and survival. Int J Radiat Oncol Biol Phys 42: 581-589, 1998.

9. Pirzkall A,Debus J,LohrF,Fuss M,Rhein B,Engenhart-Cabillic R and Wannenmacher M: Radiosurgery alone or in combination with whole-brain radiotherapy for brain metastases. J Clin Oncol 16: 3563-3569, 1998

10. Tsuruda JS, Kortman KE, Bradley WG, Wheeler DC, Van Dalsem W and Bradley TP: Radiation effects on cerebral white matter: MR evaluation. AJR Am J Roentgenol 149: 165-171, 1987.

11. Dooms GC, Hecht S, Brant-Zawadzki M, Berthiaume Y, Norman D and Newton TH: Brain radiation lesions: MR imaging. Radiology 158: 149-155, 1986. 
12. Jain R, Narang J, Sundgren PM, Hearshen D, Saksena S, Rock JP, Gutierrez J and Mikkelsen T: Treatment induced necrosis versus recurrent/progressing brain tumor: Going beyond the boundaries of conventional morphologic imaging. J Neurooncol 100: 17-29, 2010.

13. Kumar AJ, Leeds NE, Fuller GN, Van Tassel P, Maor MH, Sawaya RE and Levin VA: Malignant gliomas: MR imaging spectrum of radiation therapy- and chemotherapy-induced necrosis of the brain after treatment. Radiology 217: 377-384, 2000.

14. Smirniotopoulos JG, Murphy FM, Rushing EJ, Rees JH and Schroeder JW: Patterns of contrast enhancement in the brain and meninges. Radiographics 27: 525-551, 2007.

15. Alomari A, Rauch PJ, Orsaria M, Minja FJ, Chiang VL and Vortmeyer AO: Radiologic and histologic consequences of radiosurgery for brain tumors. J Neurooncol 117: 33-42, 2014.

16. Kamada K, Mastuo T, Tani M, Izumo T, Suzuki Y, Okimoto T, Hayashi N, Hyashi K and Shibata S: Effects of stereotactic radiosurgery on metastatic brain tumors of various histopathologies. Neuropathology 21: 307-314, 2001

17. Hirato M, Hirato J, Zama A, Inoue $H$, Ohye $C$, Shibazaki $T$ and Andou Y: Radiobiological effects of gamma knife radiosurgery on brain tumors studied in autopsy and surgical specimens. Stereotact Funct Neurosurg 66 (Suppl 1): S4-S16, 1996

18. Truong MT, St Clair EG, Donahue BR, Rush SC, Miller DC, Formenti SC, Knopp EA, Han K and Golfinos JG: Results of surgical resection for progression of brain metastases previously treated by gamma knife radiosurgery. Neurosurgery 59: 86-97, 2006.

19. Tsao MN, Rades D, Wirth A, Lo SS, Danielson BL, Gaspar LE, Sperduto PW, Vogelbaum MA, Radawski JD, Wang JZ, et al Radiotherapeutic and surgical management for newly diagnosed brain metastasis(es): An American Society for Radiation Oncology evidence-based guideline. Pract Radiat Oncol 2: $210-225,2012$
20. Sage MR, Wilson AJ and Scroop R: Contrast media and the brain. The basis of CT and MR imaging enhancement. Neuroimaging Clin N Am 8: 695-707, 1998.

21. Provenzale JM, Mukundan S and Dewhirst M: The role of blood-brain barrier permeability in brain tumor imaging and therapeutics. AJR Am J Roentgenol 185: 763-767, 2005.

22. Bucci L, Giannini A, Bellotti R, Castellano AE and Carillo C: Role of hypernephroma in a case of intracerebral hemorrhage as a 1st sign of metastasis. Case report. Riv Neurol 56: 325-335, 1986.

23. Bitoh S, Hasegawa H, Ohtsuki H, Obashi J, Fujiwara M and Sakurai M: Cerebral neoplasms initially presenting with massive intracerebral hemorrhage. Surg Neurol 22: 57-62, 1984.

24. Hernandez L, Zamorano L, Sloan A, Fontanesi J, Lo S, Levin K, Li Q and Diaz F: Gamma knife radiosurgery for renal cell carcinoma brain metastases. J Neurosurg 97 (5 Suppl): S489-S493, 2002.

25. Huber PE, Hawighorst H, Fuss M, van Kaick G, Wannenmacher MF and Debus J: Transient enlargement of contrast uptake on MRI after linear accelerator (linac) stereotactic radiosurgery for brain metastases. Int J Radiat Oncol Biol Phys 49: 1339-1349, 2001.

26. Peterson AM, Meltzer CC, Evanson EJ, Flickinger JC and Kondziolka D: MR imaging response of brain metastases after gamma knife stereotactic radiosurgery. Radiology 211: 807-814, 1999.

27. Park KR, Monsky WL, Lee CG, Song CH, Kim DH, Jain RK and Fukumura D: Mast cells contribute to radiation-induced vascular hyperpermeability. Radiat Res 185: 182-189, 2016.

28. Da Silva AN, Nagayama K, Schlesinger D and Sheehan JP: Early brain tumor metastasis reduction following Gamma Knife surgery. J Neurosurg 110: 547-552, 2009. 